\title{
A theoretical verification of intensity of plasmaspheric ELF hiss emissions: theory versus GEOS-1 observations
}

\author{
Ram Prakash ${ }^{1}$, R. P. Singh ${ }^{1}$, R. C. Awasthi ${ }^{1}$, D. P. Singh ${ }^{2}$ \\ ${ }_{1}^{1}$ Department of Physics, Bipin Bihari PG College, Jhansi-284 001, India \\ 2 Department of Physics, Government College, Adampur-125 052, Hissar, India
}

Received: 22 May 1996 / Revised: 24 January 1997 / Accepted: 28 January 1997

\begin{abstract}
An attempt is made to confirm the generation mechanism of plasmaspheric ELF hiss emissions observed aboard GEOS-1 satellite in the equatorial region both at small and large wave normal angles by calculating their magnetic field intensities in terms of incoherent Cerenkov radiation mechanism and cyclotron resonance instability mechanism, using appropriate and suitable plasma parameters. The ELF intensities calculated by Cerenkov radiation mechanism, being 4 to 5 orders of magnitude lower than the observed intensities, rule out the possibility of their generation by this mechanism. On the other hand, the intensities calculated under electron cyclotron resonance instability mechanism are found to be large enough to account for both the observed intensity and propagation losses and hence to confirm that plasmaspheric ELF hiss emissions observed aboard GEOS-1 satellite both at small and large wave normal angles were originally generated in the equatorial region by this mechanism just near the inner edge of the plasmapause. The difference in the observed intensities of two types of the emissions has been attributed to the propagation effect rather than the generation efffect.
\end{abstract}

\section{Introduction}

Broad-band structureless extremely low frequency (ELF) electromagnetic whistler-mode waves that are almost always present and commonly observed within the plasmasphere (Taylor and Gurnett, 1968; Russell et al., 1969; Muzzio and Angerami 1972; Thorne et al., 1973; Tsurutani et al., 1975; Hayakawa and Tanaka, 1977; Cornilleau-Wehrlin et al., 1978, 1993) are known as plasmaspheric hiss emissions. These emissions are

Correspondence to: Ram Prakash different from those observed in detached plasma regions of the magnetosphere (Chan et al., 1974; Hayakawa et al., 1986) and play an important role in the formation of the slot region between the inner and outer radiation belts (Lyons et al., 1972; Lyons and Thorne, 1973). Recently, Hayakawa et al. (1987) have carried out direction-finding studies for plasmaspheric ELF hiss emissions and determined the wave distribution functions of the emissions at the equatorial region inside the plasmapause, by applying the maximum entropy method to the data observed by GEOS-1 satellite. They have found that just inside the plasmapause, the wave normal direction of ELF hiss emissions was nearly aligned with the magnetic field, and when the observing position was $0.3-0.5 \mathrm{Re}$ inside of the plasmapause, there were two different groups of wave normal angles; one of medium wave normal angles ranging from $20-60^{\circ}$, and the other of large wave normal angles ranging from $70-80^{\circ}$. Hayakawa et al. (1987) have suggested that the emissions observed with small wave normal angles are generated by the electron cyclotron instability as that discussed by Thorne et al. (1973) and observed with large wave normal angles might be generated by Cerenkov or Landau resonance mechanism. On the other hand, it is widely accepted that plasmaspheric hiss emissions are generated by an anisotropic electron cyclotron resonance instability mechanism in the equatorial region (Church and Thorne, 1983; Huang et al., 1983; Cornilleau-Wehrlin et al., 1993) and incoherent Cerenkov mechanism does not contribute substantially to their generation due to Landau resonant damping at large wave normal angles (Church and Thorne, 1983; Thorne and Horne, 1994). Church and Thorne (1983) and Huang et al. (1983) have described the recent theoretical framework for electron cyclotron instability with small wave normal angles which is generally applied to plasmaspheric hiss observations.

In this study, we therefore make an attempt to confirm the generation mechanism of the plasmaspheric ELF hiss emissions observed aboard GEOS- 1 satellite in 
the equatorial region by calculating the equatorial magnetic field intensities of these emissions in terms of incoherent Cerenkov radiation mechanism and cyclotron resonance instability mechanism using appropriate and suitable plasma parameters. The intensities calculated by cyclotron resonance instability mechanism only are found to be consistent with the observed intensities. This result confirms the generation of these emissions by electron cyclotron resonance instability mechanism and rules out the possibility of their generation by incoherent Cerenkov radiation mechanism.

\section{Theoretical considerations and expressions used}

In order to calculate the intensity of plasmaspheric ELF hiss observed at large wave normal angles (oblique wave normals), we consider the expression derived by Mansfield (1964). This has been used previously by Jorgensen (1968), Lim and Laaspere (1972) and Taylor and Shawhan (1974) to interpret the auroral latitude emissions and by Prakash et al. (1979) and Prakash and Singh (1981) to interpret low-latitude VLF hiss emissions.

The Cerenkov condition in a magnetoplasma for emission of a wave at an angle $\theta$ to the magnetic field direction is

$V_{\|} \cos \theta=c / n$.

For condition of $\omega_{p}, \Omega e \gg \omega$ and $\Omega e \cos \theta \gg \omega$, the whistler mode refractive index $n$ can be given by the standard quasi-longitudinal approximation (Helliwell, 1965)

$n^{2}=\frac{\omega_{p}^{2}}{\omega \Omega e \cos \theta}$

which also requires that the wave frequency must exceed the lower hybrid frequency.

From Eqs. (1) and (2), the frequency of whistlermode wave at Cerenkov resonance can be written as (Ondoh, 1990)

$f(\mathrm{~Hz})=\frac{\omega_{p}^{2} W \cos \theta}{500 \Pi \Omega e}$

in which $W(\mathrm{keV})=250 V_{\|}^{2} / c^{2}$ is the energy of an electron with parallel velocity $V_{\|}$. The value of $\theta$ determined from Eq. (1) is substituted in the expressions of $T_{11}, T_{13}$ and $T_{33}$ which appear in the power expression of Mansfield (1964).

To calculate the magnetic field intensity in terms of electron cyclotron instability mechanism, we follow Sazhin (1984). The maximum power spectral density $\left\langle B_{f}^{2}\right\rangle_{\max }$ at the peak frequency $f_{\max }$ can be estimated from the relation (Sazhin, 1984):

$\left\langle B_{f}^{2}\right\rangle_{\max }=5 \times 10^{-17} \Lambda W_{0}^{1.5} L_{0}^{4.5} \frac{\mathrm{d} n_{i}}{\mathrm{~d} t}$

where $\Lambda=n_{e q} L^{5.5}, \frac{\mathrm{d} n_{i}}{\mathrm{~d} t}$ is the rate of influx of electrons, $W_{0}$, the electron energy at $L=L_{0}$ in $\mathrm{eV}$, and $L_{0}$ can be chosen arbitrarily. This relation gives intensity directly in $\gamma^{2} \mathrm{~Hz}^{-1}$ in the equatorial plane. Equation (4) can very well be applied to account for the intensity of plasmaspheric ELF hiss emissions generated in the equatorial region at small wave normal angles (Sazhin, 1989; Hayakawa and Sazhin, 1992). The frequency $f_{\max }$ corresponding to peak power spectral density can be calculated using the relation (Hayakawa et al., 1988):

$f_{\max }=\frac{1.6 \times 10^{9}}{L^{9} n_{e q} W}$

in which $n_{e q}$ is the equatorial electron density in $\mathrm{cm}^{-3}$ and $W$ is the characteristic parallel energy of the incoming electrons in $\mathrm{keV}$.

The gain in wave instability due to electron cyclotron instability in the equatorial region is given by (Sazhin, 1984, 1989):

$G=2 \int_{- \text {Sion }}^{\text {Sion }} \frac{\gamma(s) \mathrm{d} s}{V_{g}(s)}=\log _{e}\left(\frac{1}{R}\right)$

where $R$ is the wave energy reflection coefficient, which is frequency dependent in general. This relation expresses the stationary character of the process of wave generation as achieved by the balance of total wave amplification along the field-line and its reflection from the ionosphere.

The gain in decibels can be calculated using the relation (Church and Thorne, 1983)

Gain $(\mathrm{d} B)=10 \log \left(e^{G}\right)$

\section{Results and discussion}

Figure 1 shows the variation of the experimentally observed magnetic field intensity of plasmaspheric ELF hiss emissions with the wave frequency for three equatorial observations of August 5, September 4 and September 28, 1977 on board GEOS-1 satellite. Figure 1 is reproduced from Fig. 2 of Hayakawa et al. (1987). Hereafter, we shall refer the emissions of 5th August as type I emissions and those of September 4 and 28 as type II emissions. Type I emissions exhibit a rather flat distribution in a frequency range of $300-450 \mathrm{~Hz}$. with a peak intensity of $1.44 \times 10^{-4} \gamma^{2} \mathrm{~Hz}^{-1}$ at $350 \mathrm{~Hz}$. Direction finding results show that these emissions are observed at the inner edge of the plasmapause and have quasi-longitudinal wave normals $\left(\theta \approx 5-15^{\circ}\right.$ ) (Hayakawa et al., 1987). Quasi-linear electron cyclotron instability (Sazhin, 1984; Hayakawa et al., 1986; Church and Thorne, 1983) can be held responsible for the generation of such type of emissions. Type II emissions have a peak intensity of $\sim 6-9 \times 10^{-6} \gamma^{2} \mathrm{~Hz}^{-1}$. Type II emissions of 4th September 1977 have a secondary peak also with an intensity of $1.33 \times 10^{-6} \gamma^{2} \mathrm{~Hz}^{-1}$ at $\sim 550 \mathrm{~Hz}$. Such double peaks observed in the intensity spectra of DP hiss emissions have been satisfactorily explained (Prakash and Singh, 1994) in terms of quasilinear electron cyclotron instability mechanism proposed by Sazhin (1984). The similarity of primary peaks 


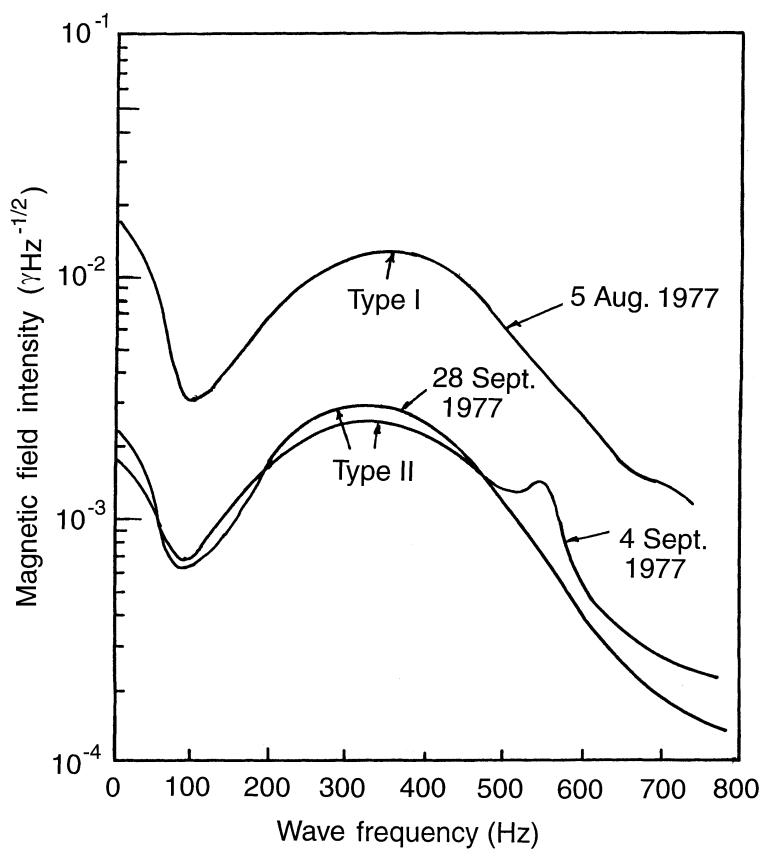

Fig. 1. Magnetic field intensity versus frequency curves of plasmaspheric ELF hiss emissions as observed by GEOS-1 satellite (after Hayakawa et al., 1987)

in the intensity spectra of type I and type II emissions in Fig. 1 indicates that both types of emissions should be generated by the same mechanism. However, type II emissions have large wave normal angles ranging from $20^{\circ}$ to $80^{\circ}$ which led Hayakawa et al. (1987) to suggest that these emissions might be generated by the Cerenkov or Landau resonance mechanism.

In order to confirm the generation mechanism of plasmaspheric ELF hiss emissions with large wave normal angles (oblique wave normals) observed aboard GEOS-1 satellite, we first consider incoherent Cerenkov radiation mechanism and calculate the frequencies of the whistler-mode waves at Cerenkov resonance at different values of the wave normal angle $\theta$ by using Eq. (3) and assuming their generation at $L=4.8$ and 5.5, just near the inner edge of the plasmapause in the equatorial plane. The plasmapause is located at $L=5.8$ on 4 th September and at $L \sim 5$ on 28 th September, 1977 (Hayakawa et al., 1987). The electron densities at the $L$-values of 4.8 and 5.5 are taken to be 200 and 100 el. $\mathrm{cm}^{-3}$ respectively which roughly correspond to the diffusive equilibrium model (Angerami and Thomas, 1963). The plasma frequency is calculated using the relation $\omega_{p} / 2 \Pi(\mathrm{kHz})=8.98 n_{\mathrm{o}}^{1 / 2}$ where $n_{\mathrm{o}}$ is in el. $\mathrm{cm}^{-3}$ and the electron gyrofrequency $\Omega e$ is calculated from the relation $\Omega e / 2 \Pi(\mathrm{kHz})=873.6 / L^{3}$. The frequencies of whistler-mode waves at Cerenkov resonance calculated from Eq. (3) at the $L$-values of 4.8 and 5.5 are plotted against wave normal angle $\theta$ in Fig. 2. The energies of the resonant electrons used in the calculations are also indicated in this figure. The calculated frequency spectrum of whistler-mode waves is in good agreement with the observed frequency spectrum of Fig. 1. Figure 2

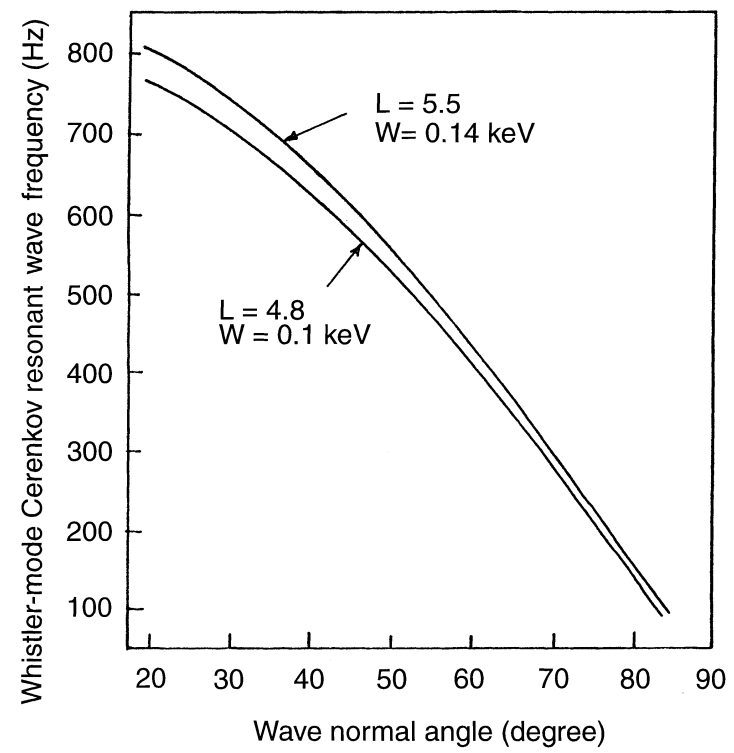

Fig. 2. Plots showing variation of whistler-mode Cerenkov resonant wave frequency with wave normal angle

depicts that higher frequency components may be radiated out at lower values of wave normal angle.

Now, we calculate the magnetic field intensity of plasmaspheric ELF hiss in $W \times \mathrm{Hz}^{-1}$ per electron at the $L$-values of 4.8 and 5.5 in the equatorial plane by considering incoherent Cerenkov radiation mechanism. The wave frequency is taken to be $350 \mathrm{~Hz}$ at which the maximum intensity of type II emissions has been observed (Hayakawa et al., 1987). The pitch angle of radiating electrons is assumed to be zero because such electrons give rise to emissions of higher intensity (Prakash et al., 1979). The energies of the electrons are taken to be 0.1 and $0.14 \mathrm{keV}$ because with these energies the calculated frequency spectrum of whistler-mode waves at Cerenkov resonance is found to be in good agreement with the observed frequency spectrum and also higher intensities are radiated out. The calculated equatorial intensities at the $L$-values of 4.8 and 5.5 for $350 \mathrm{~Hz}$ waves are found to be $2.5732 \times 10^{-33}$ and $2.3674 \times 10^{-32} \mathrm{~W} \mathrm{~Hz}^{-1}$ per electron respectively. We calculated the volumes of the flux tubes along the field lines corresponding to $L=4.8$ and 5.5 , each of which has a cross section of $1 \mathrm{~cm}^{2}$ at $500 \mathrm{~km}$ in the ionosphere and extends to the equatorial plane. The volumes of these flux tubes are found to be $2.2520 \times 10^{11} \mathrm{~cm}^{3}$ and $3.9217 \times 10^{11} \mathrm{~cm}^{3}$ respectively. Now, we have to consider a suitable energy spectra for the particles used in the present calculations so as to determine their densities in the flux tubes. The energy spectra of $\sim 10^{5} \mathrm{el} . \mathrm{cm}^{-2} \mathrm{~s}^{-1}$ sterad $^{-1}$ observed aboard Hitch-hiker 1 (1963-25 B) satellite for outer belt electrons (Katz, 1966) is unsuitable for present calculations because these electrons have energy $\geq 1 \mathrm{MeV}$. O'Brien (1966) has reported a maximum flux of $\sim 5 \times 10^{6}$ el. $\mathrm{cm}^{-2} \mathrm{~s}^{-1} \mathrm{sterad}^{-1}$ for energetic electrons of energy $>40 \mathrm{keV}$ in the region of $L$-values of 4.8-5.5. Similarly, Cornilleau-Wehrlin et al. 
(1993) have reported the differential flux intensity for $25 \mathrm{keV}$ electrons in the pitch angle range of $85^{\circ}<\alpha<95^{\circ}$ to be between $2.2 \times 10^{5}$ and $1.7 \times 10^{7}$ $\left(\mathrm{cm}^{2} \mathrm{~s} \text { sterad } \mathrm{keV}\right)^{-1}$. Since strongest hiss events correspond to largest electron fluxes and the observed flux at lower energies is always higher than that at higher energies (Katz, 1966; Cornilleau-Wehrlin et al., 1993), we consider a flux of $5 \times 10^{8}$ el. $\mathrm{cm}^{-2} \mathrm{~s}^{-1}$ sterad $^{-1}$ at the $L$-values under consideration for 0.1 and $0.14 \mathrm{keV}$ electrons. From this flux value, we find the densities of 0.1 and $0.14 \mathrm{keV}$ electrons in the flux tubes along the field lines corresponding to the $L$-values of 4.8 and 5.5 to be 10.17 and $8.85 \mathrm{~cm}^{-3}$, respectively. We further assume that the flux remains constant along the field lines under consideration and multiply these densities with the tube volumes and the intensities calculated in the equatorial plane to obtain intensities in $\mathrm{W} \mathrm{m} \mathrm{m}^{-2} \mathrm{~Hz}^{-1}$ there. The calculated equatorial intensities at the $L$-values of 4.8 and 5.5 are found to be $6.05 \times 10^{-17}$ and $8.22 \times 10^{-16} \mathrm{~W}$ $\mathrm{m}^{-2} \mathrm{~Hz}^{-1}$ respectively which are equivalent to $1.98 \times 10^{-11}$ and $2.36 \times 10^{-10} \gamma^{2} \mathrm{~Hz}^{-1}$ respectively. These values are 4 to 5 orders of magnitude lower than the observed intensities of type II emissions. Although incoherent Cerenkov mechanism radiates out waves at large wave normal angles near the resonance cone, the radiated intensities, being 4 to 5 orders of magnitude lower than the observed intensities, rule out the possibility of generation of oblique wave normals in this mechanism. Further, the condition of quasi-longitudinal approximation that the wave frequency must exceed the lower hybrid frequency is not always valid for plasmaspheric hiss and the condition $\Omega e \cos \theta \gg \omega$ assumed in the derivation of Eq. (3) which has been used in the calculation of the frequency spectrum of the radiated whistler-mode waves, is not rigorously true for all values of $\theta$ at $L=4.8$ and 5.5. Thus type II emissions cannot be generated by the Cerenkov mechanism. This negative result is also in coherence with the well-known fact that the incoherent Cerenkov mechanism does not contribute substantially to the generation of plasmaspheric hiss because at large wave normal angles, waves are usually subject to Landau resonant damping (Church and Thorne, 1983; Thorne and Horne, 1994) with superthermal electrons (100 eV $\leq \mathrm{E} \leq \mathrm{keV})$.

We now assume that type I emissions of August 5, 1977 are generated in the equatorial region at $L=5.9$ near the inner edge of the plasmapause (relatively sharp plasmapause located at $L_{p p}=5.50-6.20$ ) and type II emissions of September 4 and September 28, 1977 are generated at the equatorial locations of $L=5.5$ (very sharp plasmapause at $L_{p p}=5.8$ ) and 4.8 (very gradual gradient, plasmapause location $L_{p p}=5.0$ ) respectively at small wave normal angles $\left(\theta \sim 0^{\circ}\right)$ under electron cyclotron resonance instability mechanism (Sazhin, 1984). To calculate the intensity of these emissions, we first determine the characteristic energy of the energetic electrons likely to be responsible for generation of these emissions. From Eq. (5), the characteristic energies of the energetic electrons for $f_{\max }=350 \mathrm{~Hz}$ are estimated to be within $\sim 10-20 \mathrm{keV}$ range. The electron density at $L=5.9$ is estimated to be $50 \mathrm{~cm}^{-3}$ from Fig. 1 of Parrot and Lefeuvre (1986) which is based on the electron density measurements performed by the S-304 mutual impedance technique on board GEOS-1 satellite. In the present calculations, the value of $\mathrm{d} n_{i} / \mathrm{d} t$ is assumed to be $3 \times 10^{-4} \mathrm{~cm}^{-3} \mathrm{~s}^{-1}$ at all the three $L$ - values under consideration which is the same as adopted by Sazhin (1984) at $L=3.6$ and Nishino and Tanaka (1994) at $L=4.8$ in the study of plasmaspheric ELF/VLF hiss emissions. The calculated values of the characteristic energy are used to estimate the peak power spectral densities from equation (4) for $f_{\max }=350 \mathrm{~Hz}$ at the three equatorial locations of $L=4.8,5.5$ and 5.9. The calculated and observed intensities alongwith other parameters are presented in Table 1 .

Table 1 shows that the calculated values of intensity at the $L$-values of 4.8 and 5.5 are greater than the observed intensities by about 7 and $8 \mathrm{~dB}$ respectively. However, the calculated intensity at $L=5.9$ is lower than the observed intensity by about $5 \mathrm{~dB}$. Since the properties of hiss are strongly influenced by wave propagation characteristics (Thorne et al., 1979; Church and Thorne, 1983), the difference in the calculated and observed intensities may be due to the propagation effect. Type I emissions which have quasi-longitudinal wave normals $\left(\theta \approx 5-15^{\circ}\right)$ at the observing site just at the inner edge of the plasmapause (Hayakawa et al., 1987) might have crossed the equatorial source region at least once nearly aligned with the Earth's magnetic field between the times of their generation and observation similar to the "cyclic waves" discussed by Thorne et al. (1979) and so they are likely to be amplified further. Therefore, we calculate the power gain for $350 \mathrm{~Hz}$ waves at $L=5.9$ in the equatorial region. For this, we set $R=0.1$ (Etcheto et al., 1973; Sazhin, 1984) at $350 \mathrm{~Hz}$ in Eq. (6) which gives $G=2.3026$ and hence Eq. (7) calculates a gain of $10 \mathrm{~dB}$ in a single transit through the source region. Thus the intensity of $4.1563 \times 10^{-5} \gamma^{2}$ $\mathrm{Hz}^{-1}$ initially radiated out at $L=5.9$ in electron cyclotron instability now becomes $4.1563 \times 10^{-4} \gamma^{2}$ $\mathrm{Hz}^{-1}$ which is greater than the observed value of $1.44 \times 10^{-4} \gamma^{2} \mathrm{~Hz}^{-1}$ by $4.60 \mathrm{~dB}$. The excess intensity by $4.60 \mathrm{~dB}$ may very well account for the propagation loss suffered by these waves during the period of their
Table 1. Observed and calculated equatorial intensities with other parameters used

\begin{tabular}{llllll}
\hline$L=L_{0}$ & $W(\mathrm{keV})$ & $n_{\text {eq }}\left(\mathrm{cm}^{-3}\right)$ & $\Lambda\left(\mathrm{cm}^{-3}\right)$ & \multicolumn{2}{l}{ Intensity in $\gamma^{2} \mathrm{~Hz}^{-1}$} \\
\cline { 4 - 5 } & & & Calculated & Observed \\
\hline 4.8 & 16.90 & 200 & $1.1165 \times 10^{6}$ & $4.2787 \times 10^{-5}$ & $9.00 \times 10^{-6}$ \\
5.5 & 9.93 & 100 & $1.1803 \times 10^{6}$ & $3.7575 \times 10^{-5}$ & $6.25 \times 10^{-6}$ \\
5.9 & 10.35 & 50 & $3.6827 \times 10^{5}$ & $4.1563 \times 10^{-5}$ & $1.44 \times 10^{-4}$ \\
\hline
\end{tabular}


generation and observation aboard GEOS-1 satellite just inside the plasmapause. Thus we find that type I emissions could very well be generated and maintained by the anisotropic electron cyclotron instability mechanism just near the inner edge of the plasmapause (Hayakawa et al., 1987; Church and Thorne, 1983). It may be pointed out that the observed intensity of type I emissions shown in Fig. 1 is extraordinarily greater than the average hiss intensities presented recently by Cornilleau-Wehrlin et al. (1993) who have found that energetic electron differential fluxes around the magnetic equator are large enough to allow wave amplification upto an intensity of $\sim 1 \times 10^{-6} \gamma^{2} \mathrm{~Hz}^{-1}$ within $10^{\circ}$ of latitude around the geomagnetic equator in a single crossing of the waves through this region.

Type II emissions which have oblique wave normals $\left(\theta \approx 20-80^{\circ}\right)$ at the observing site that is $0.3-0.5 \mathrm{Re}$ inside from the plasmapause (Hayakawa et al., 1987) do not pass through the equatorial amplification region before their observation due to their large wave normal angles and hence are not amplified further. Instead, they suffer comparatively greater propagation losses including the losses due to Landau damping and collisional as well as spreading losses (Taylor and Shawhan, 1974). The difference of 7-8 $\mathrm{dB}$ between calculated and observed intensities of type II emissions seems to be sufficient to account for such losses (Helliwell, 1965).

Aikyo and Ondoh (1971) and Huang and Goertz (1983) have shown by ray-tracing studies that nonducted waves launched longitudinally at the geomagnetic equator become oblique as they propagate away from it. The wave normals are tilted towards the earth by decreasing plasma density with increasing $L$-value and outward from the Earth by decreasing magnetic field strength with increasing $L$-value, jointly with the effect of field line curvature. The two competing effects are unlikely to cancel each other exactly as one may dominate over the other. Therefore, as the wave propagates, its normal becomes more and more inclined to the field and ultimately approaches the resonance cone. With a suitable distribution of the plasma in the magnetosphere, it might be possible that type II emissions propagating in this way were magnetospherically reflected back and reached the GEOS-1 satellite with large wave normal angles. Thus, it seems to us that like type I emissions type II emissions were also generated by the electron cyclotron instability mechanism.

In the light of these results we finally confirm that both type I and type II plasmaspheric ELF hiss emissions observed by GEOS-1 satellite were originated by electron cyclotron resonance instability mechanism and that the difference in their intensities is due to propagation effect. By three dimensional ray path calculations of the integrated gain in a realistic model plasmasphere, Church and Thorne (1983) have also confirmed the anisotropic electron cyclotron resonant generation mechanism for the maintenance of hiss throughout the magnetosphere.

\section{Conclusion}

In this study we have carried out detailed intensity calculations for the plasmaspheric ELF hiss emissions observed aboard GEOS-1 satellite both at small (type I emissions) and large (type II emissions) wave normal angles by adopting suitable plasma parameters and considering the incoherent Cenenkov radiation process and cyclotron resonance instability as their generation mechanisms. The intensities calculated by the Cerenkov radiation mechanism are 4 to 5 orders of magnitude lower than the observed intensities and thus rule out the generation of oblique wave normals by this mechanism. On allowing appropriate propagation losses, the intensities calculated by the electron cyclotron instability mechanism are found to be quite consistent with the observed intensities. It is, therefore, confirmed that the plasmaspheric ELF hiss emissions observed aboard GEOS-1 satellite both at small and large wave normal angles were generated by the same electron cyclotron instability mechanism just near the inner edge of the plasmapause, the difference in observed intensities of the two types of emissions being attributed to the propagation effect rather than the generation effect.

Acknowledgements The authors are grateful to the Principals of their respective colleges for providing them necessary research facilities. One of the authors (DPS) is grateful to the University Grants Commission, New Delhi for providing financial support in the form of a minor project. The authors also wish to thank the referees for helpful comments and suggestions.

Topical Editor K.-H. Glaßmeier thanks S. S. Sazhin and another referee for their help in evaluating this paper.

\section{References}

Aikyo, K., and T. Ondoh, Propagation of non-ducted VLF waves in the vicinity of the plasmapause, J.Radio Res. Labs., Japan, 18, 153,1971

Angerami, J. J., and J. O. Thomas, The distribution of ions and electrons in the Earth's exosphere, Techn. Rep. 3412-3, Stanford University, CA, 1963.

Chan, K. W., R. E. Holzer, and E. J. Smith, A relation between ELF hiss and plasma density in the outer plasmasphere, J. Geophys. Res., 79, 1989, 1974.

Church, S. R., and R. M. Thorne, On the origin of plasmaspheric hiss; ray path integrated amplification, J. Geophys. Res., 88, 7941, 1983.

Cornilleau-Wehrlin, N., R. Gendrin, F. Lefeuvre, M. Parrot, R. Grard, D. Jones, A. Bahnson, E. Ungstrup, and W. Gibbons, VLF electromagnetic waves observed on board GEOS-1, Space Sci. Rev., 22, 371, 1978.

Cornilleau-Wehrlin, N., J. Solomon, A. Korth, and G. Kremser, Generation mechanism of plasmaspheric hiss: a statistical study from GEOS-1 data, J. Geophys. Res., 98, 21471, 1993.

Etcheto, J., R. Gendrin, J. Solomon, and A. Roux, A self consistent theory of magneto spheric ELF hiss, J. Geophys. Res., 78, 8150, 1973.

Hayakawa, M., and S. S. Sazhin, Mid-latitude and plasmaspheric hiss: a review, Planet. Space Sci., 40, 1325, 1992.

Hayakawa, M., N. Ohmi, M. Parrot, and F. Lefeuvre, Direction finding of ELF hiss emissions in a detached plasma region of the magnetosphere, J. Geophys. Res., 91, 135, 1986.

Hayakawa, M., M. Parrot, and F. Lefeuvre, The wave distribution functions of plasmaspheric ELF hiss: GEOS-1 observation in equatorial region, Mem. Natn. Inst. Polar Res., 47, 157, 1987. 
Hayakawa, M., and Y. Tanaka, ELF emissions observed at Moshirl, Nature, 270, 703, 1977.

Hayakawa, M., Y. Tanaka, S. S. Sazhin, M. Tixier, and T. Okada, Substorm associated VLF emissions with frequency drift observed in the premidnight sector, J. Geophys. Res., 93, $5685,1988$.

Helliwell, R. A., Whistlers and related ionospheric phenomena, Stanford University Press, Stanford, CA, 1965.

Huang, C. Y., and C. K. Goertz, Ray-tracing studies and pathintegrated gains of ELF unducted whistler-mode waves in the earth's magnetosphere, J. Geophys. Res., 88, 6181, 1983.

Huang, C. Y., C. K. Goertz, and R. R. Anderson, A theoretical study of plasmaspheric hiss generation, J. Geophys. Res., 88, 7928, 1983.

Jorgenson, T. S., Interpretation of auroral hiss measured on OGO2 and at Byrd Station in terms of Cerenkov radiation, J. Geophys. Res., 73, 1055, 1968.

Katz, L., Electron and proton observations, in radiation trapped in the Earth's magnetic fields, p 129, Reidel, Holland, 1966.

Lim, T. L., and T. Laaspere, An evaluation of the intensity of Cerenkov radiation from auroral electrons with energies down to $100 \mathrm{eV}$, J. Geophys. Res., 77, 4145, 1972.

Lyons, L. R., and R. M. Thorne, Equilibrium structure of radiation belt electrons, J. Geophys. Res., 78, 2142, 1973.

Lyons, L. R., R. M. Thorne, and C. F. Kennel, Pitch angle diffusion of radiation belt electrons within the plasmasphere, J. Geophys. Res., 77, 3455, 1972.

Mansfield, V. N., Cerenkov and cyclotron radiation as VLF emission sources, Radiophysics Lab., Thayer School of Engineering, Hanover, New Hampshire, 1964.

Muzzio, J. L. R., and J. J. Angerami, OGO-4 observations of extremely low frequency hiss, J. Geophys. Res., 77, 1157, 1972.

Nishino, M., and Y. Tanaka, Low-latitude VLF hiss associated with the severe magnetic storm on October 19-21, 1989, J. Geomagn. Geoelect., 46, 193, 1994.

O' Brien, B. J., Precipitation of electrons and protons into the atmosphere in radiation Trapped in the Earth's magnetic field, p 321, Reidel, Holland, 1966.
Ondoh, T., Broad-band auroral VLF hiss and inverted electron precipitation in the polar magnetosphere, J. Atmos. Terr. Phys., 52, 385, 1990.

Parrot, M., and F. Lefeuvre, Statistical study of the propagation characteristics of ELF hiss observed on GEOS-1, inside and outside the plasmapause, Ann. Geophysicae, 4, 363, 1986.

Prakash, R., and B. Singh, A theoretical verification of the intensity of VLF emissions observed by Ariel $3 / 4$ satellites, Ann. Geophysicae, 37, 557, 1981.

Prakash, R., and R. P. Singh, Generation mechanism of ELF hiss emissions in the detached plasma regions of magnetosphere, Indian J. Radio Space Phys., 23, 284, 1994.

Prakash, R., D. P. Singh, and B. Singh, Incoherent Cerenkov process as a source of low latitude VLF emissions, Planet. Space Sci., 27, 959, 1979.

Russell, C. T., R. E. Holzer, and E. J. Smith, Observations of ELF noise in the magnetosphere, I. Spatial extent and frequency of occurrence, J. Geophys. Res., 74, 755, 1969.

Sazhin, S. S., A model for hiss-type mid-latitude VLF emissions, Planet. Space Sci., 32, 1263, 1984.

Sazhin, S. S., Improved quasilinear models of parallel whistlermode instability, Planet. Space Sci., 37, 633, 1989.

Taylor, W. W. L., and D. A. Gurnett, Morphology of VLF emissions observed with the Injun 3 satellite, J. Geophys. Res., 73, 5615, 1968.

Taylor, W. W. L., and S. D. Shawhan, A test of incoherent Cerenkov radiation for VLF hiss and other magnetospheric emissions, J. Geophys. Res., 79, 105, 1974.

Thorne, R. M., and R. B. Horne, Landau damping of magnetospherically reflected whistlers, J. Geophys. Res., 99, 17249, 1994.

Thorne, R. M., E. J. Smith, R. K. Burton, and R. E. Holzer, Plasmaspheric hiss, J. Geophys. Res., 78, 1581, 1973.

Thorne, R. M., S. R. Church, and D. J. Gorney, On the origin of plasmaspheric hiss: the importance of wave propagation and the plasmapause, J. Geophys. Res., 84, 5241, 1979.

Tsurutani, B. T., E. J. Smith, and R. M. Thorne, Electromagnetic hiss and relativistic electron losses in the inner zone, J. Geophys. Res., 80, 600, 1975. 